\title{
Knee Angle Estimation Algorithm for Myoelectric Control of Active Transfemoral Prostheses
}

\author{
Alberto López Delis ${ }^{1,2}$, João Luiz Azevedo de Carvalho ${ }^{1,3}$, Adson Ferreira da Rocha ${ }^{1}$, \\ Francisco Assis de Oliveira Nascimento ${ }^{1}$, and Geovany Araújo Borges ${ }^{1}$ \\ ${ }^{1}$ Department of Electrical Engineering, University of Brasília, Brasília-DF, Brazil \\ lopez_delis@yahoo.com, adson@unb.br, assis@unb.br \\ gaborges@ene.unb.br \\ ${ }^{2}$ Medical Biophysics Center, University of Oriente, Santiago de Cuba, Cuba \\ ${ }^{3}$ UnB-Gama Faculty, University of Brasília, Gama-DF, Brazil \\ joaoluiz@gmail.com
}

\begin{abstract}
This paper presents a bioinstrumentation system for the acquisition and pre-processing of surface electromyographic (SEMG) signals, and a knee angle estimation algorithm for control of active transfemoral leg prostheses, using methods for feature extraction and classification of myoelectric signal patterns. The presented microcontrolled bioinstrumentation system is capable of recording up to four SEMG channels, and one electrogoniometer channel. The proposed neural myoelectric controller algorithm is capable of predicting the intended knee joint angle from the measured SEMG signals. The algorithm is designed in three stages: feature extraction, using auto-regressive model and amplitude histogram; feature projection, using self organizing maps; and pattern classification, using a Levenberg-Marquardt neural network. The use of SEMG signals and additional mechanical information such as that provided by the electrogoniometer may improve precision in the control of leg prostheses. Preliminary results are presented.
\end{abstract}

Keywords: Electromyographic signal, prosthesis control, microcontrolled bioinstrumentation, feature extraction, dimensionality reduction, neural networks.

\section{Introduction}

The use of microprocessors in myoelectric control has grown notably, benefitting from the functionality and low cost of these devices. Microprocessors provide the ability to employ advanced signal processing and artificial intelligence methods as part of a control system, while easily conforming to control options, and adjusting to the input characteristics. They also provide the ability to implement patternrecognition-based control schemes, which increases the variety of control functions, and improves robustness.

Surface electromyographic (SEMG) signals provide a non-invasive tool for investigating the properties of skeletal muscles [1]. The bandwidth of the recorded potentials is relatively narrow $(50-500 \mathrm{~Hz})$, and their amplitude is low $(50 \mu \mathrm{V}-5 \mathrm{mV})$ [2]. 
These signals have been used not only for monitoring muscle behavior during rehabilitation programs [3], but also for mechanical control of prostheses. In this context, it is important to be able to correctly predict which movement is intended by the user. The SEMG signal is very convenient for such application, because it is noninvasive, simple to use, and intrinsically related to the user's intention. However, there are other useful variables, especially those related to proprioception, for example: the angle of a joint, the position of the limb, and the force being exerted.

This project is motivated by the ongoing development of an active leg prosthesis prototype (Fig. 1). The prosthesis has three degrees of freedom: one for the knee (sagittal plane), and two movements for the foot (sagittal and frontal planes). The three degrees of freedom are associated with the angles $\theta_{1}, \theta_{2}$ and $\theta_{3}$, controlled by DC reduction motors. The prototype will be fixed to the patient's upper leg through a fixing capsule, where the SEMG sensors will be placed. The prosthesis will receive control commands through digital signal processing, feature extraction, and pattern classification. For the development of an active leg prosthesis that also possesses ankle and foot axes, it is necessary to use other sources of information besides the SEMG signal [4]. In this context, the use of myoelectric signals combined with other variables related to proprioception may improve the reliability in closed-loop control systems. The bioinstrumentation system should also be as immune to noise and interference as possible. This can be achieved by proper board and shielding design, as well as the use of filters whenever they are necessary.

Figure 2 presents the typical main components of a general myoelectric algorithm based on pattern recognition. The SEMG signals are acquired by surface electrodes placed on the skin over antagonistic leg muscles of the subject. The signals originating from the electrodes are pre-amplified to differentiate the small signals of interest, and then are amplified, filtered and digitized. Finally, the information is transferred to a myoelectric knee angle estimation algorithm.

In the design and implementation of myoelectric control algorithms [5], the system's precision is essential for an accurate accomplishment of the user's intention. Precision is an important factor on the development of multi-sensory controllers, and can be improved by extracting more information from the muscle's state, and using a classifier that is capable of processing this information. The myoelectric control algorithm should be capable of learning the muscular activation patterns that are used

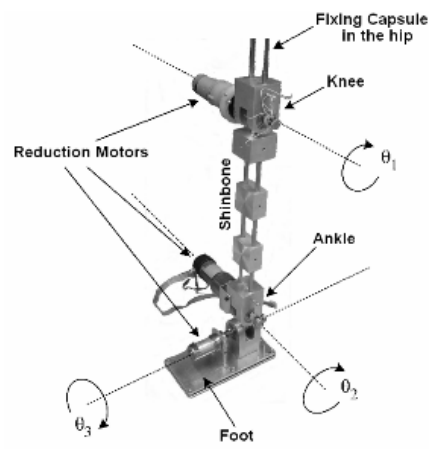

Fig. 1. Mechanical structure of the prosthesis prototype 


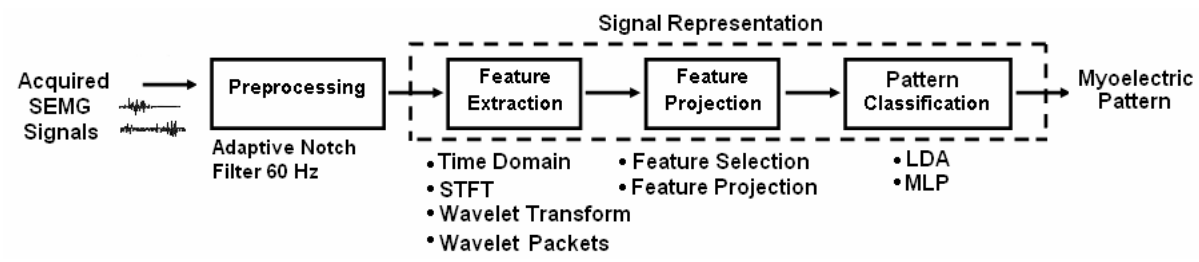

Fig. 2. Typical main components of a general myoelectric control algorithm based on pattern recognition

in natural form for typical movements. It also needs robustness against condition variations during the operation. The response time cannot create delays that are noticeable to the user.

This article presents a micro-controlled bioinstrumentation prototype system as part of the development of an active leg prosthesis structure that allows the acquisition and processing of electromyographic signals and other data related to articulate movement, specifically the angle of the knee joint. The information obtained is processed in order to obtain appropriate myoelectric patterns for prosthesis control. Preliminary results on the design of pattern recognition algorithms for the estimation of the knee joint angle are presented.

\section{Methods}

The front end stage of the designed bioinstrumentation system acquires up to four SEMG channels. The SEMG signals are measured on a pair of agonist and antagonist muscles of the leg (Figs. 3a and 3b). An electrogoniometer is used to measure the flexion and extension angles of the knee joint (Fig. 3c).
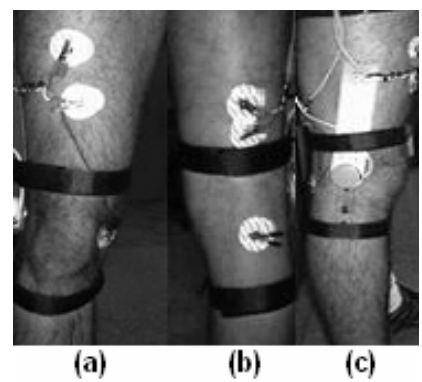

Fig. 3. Experimental setup. Surface electrodes are placed over a pair of agonist and antagonist muscle groups of the leg: (a) vastus intermedius, (b) semitendinosus. An electrogoniometer is used to measure the flexion and extension angles of the knee joint (c).

Differential amplifiers, used in the bipolar configuration, significantly reduce the common mode interference signals (CMRR $>110 \mathrm{~dB}$ ). A band-pass filter in the 20$500 \mathrm{~Hz}$ frequency range is used. It is composed by a low-pass filter and high-pass filter with a programmable gain stage based on digital potentiometers, controlled by the microcontroller. These elements allow subject-based setting of the SEMG gain 
levels. To minimize power consumption and increase noise immunity, operational amplifiers with JFET inputs were used. To obtain adequate myoelectric amplitude, an overall gain of up to 20000 can be programmed at the front end [2].

A second block, micro-controlled and optically isolated from the front end (Fig. 4), centralizes all the functions associated with the analog/digital conversion process, implementing the digital gain control for the front end amplifiers and the synchronized sampling of SEMG signals. The microcontrollers are from the ARM SAM7S64 ATMEL family of high performance processors, based on 32-bit RISC architecture with an integrated group of peripherals that minimize the number of external components.

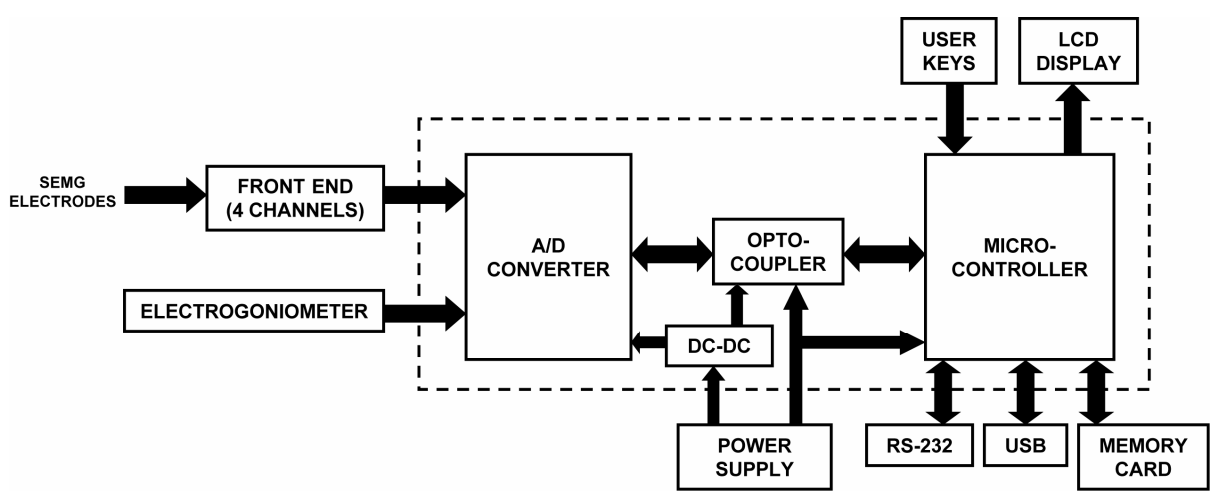

Fig. 4. Block diagram of the bioinstrumentation system

A 13-bit A/D converter with a serial peripheral interface (SPI) is used for signal sampling, and allows discriminating small amplitude levels. The electrogoniometer channel is coupled to the system, and generates an electric signal corresponding to the angular position ranging from 30 to 240 degrees. The sampling frequency of each channel is $1744.25 \mathrm{~Hz}$. Figure 5 presents example data acquired during an experimental measurement.
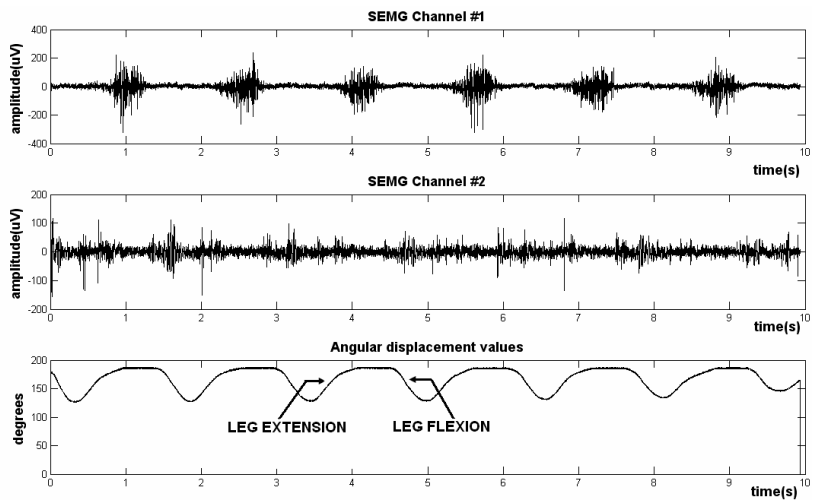

Fig. 5. Recorded SEMG signals (rectus femoris and opposite muscles) and measured knee joint angle, from a representative 10 -second experiment 
The microcontroller is linked through RS-485 protocol to the central processor of the prosthesis, which is responsible for coordinating the tasks in the control process. Besides the RS-485 protocol, which provides the interaction of the block with the central processor, RS-232C and USB interfaces are available for the communication with a PC when the system is configured in stand alone mode (Fig. 6). In this mode, the system allows the visualization of the state of the experiments during their realization using a LCD display. The instrumentation system is designed using low power consumption components, which increases the system's portability.

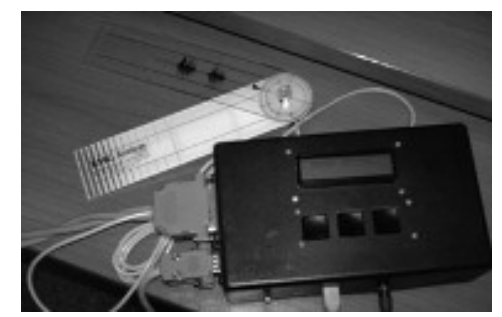

Fig. 6. Bioinstrumentation module (with electrogoniometer) configured in stand alone mode

\subsection{Adaptive Filter Implementation}

The power line interference usually has its first harmonics $(60 \mathrm{~Hz}, 120 \mathrm{~Hz}, 180 \mathrm{~Hz}$, and $240 \mathrm{~Hz}$ ) in a portion of the spectrum with major SEMG energy concentration. The use of an analog notch filter may distort the signal; therefore it should only be used when really necessary. Generally, the best option is to use an adaptive notch filter. An embedded subroutine in the SAM7S64's core implements an adaptive notch filter in real time. This filter maintains a running estimate of the $60 \mathrm{~Hz}$ interference, and the current noise at time $t$ can be estimated from the previous two noise estimates [6], as shown in equations (1) and (2),

$$
e(t)=N \cdot e(t-n T)-e(t-2 n T)
$$

where $T$ is the sample period and $N=2 \cos (2 \pi 60 T)$. In the filter, the output is generated by subtracting the estimated noise, $e(t)$, from the input signal, $x(t)$. The following expression is used to implement the filter:

$$
f(t)=[x(t)-e(t)]-[x(t-n T)-e(t-n T)] .
$$

If $f(t)>0$, then the estimate was too low, so we adjust the noise estimate upwards by incrementing by $d$ :

$$
e(n T+T)=e(n T+T)+d .
$$

If $f(t)<0$, the estimate was too high, so it is decremented by $d$ :

$$
e(n T+T)=e(n T+T)-d .
$$

Using large vales of $d$, the filter adapts more rapidly, and exhibits a broad bandwidth. For small values of $d$, the filter adapts more slowly, and has a narrower 
bandwidth. The selection of the $d$ factor is empiric, based on test realizations, and its value is small compared to the dynamic range of the A/D converter [6]. Figure 7 shows the adaptive filtering of a SEMG signal measured on the rectus femoris muscle.
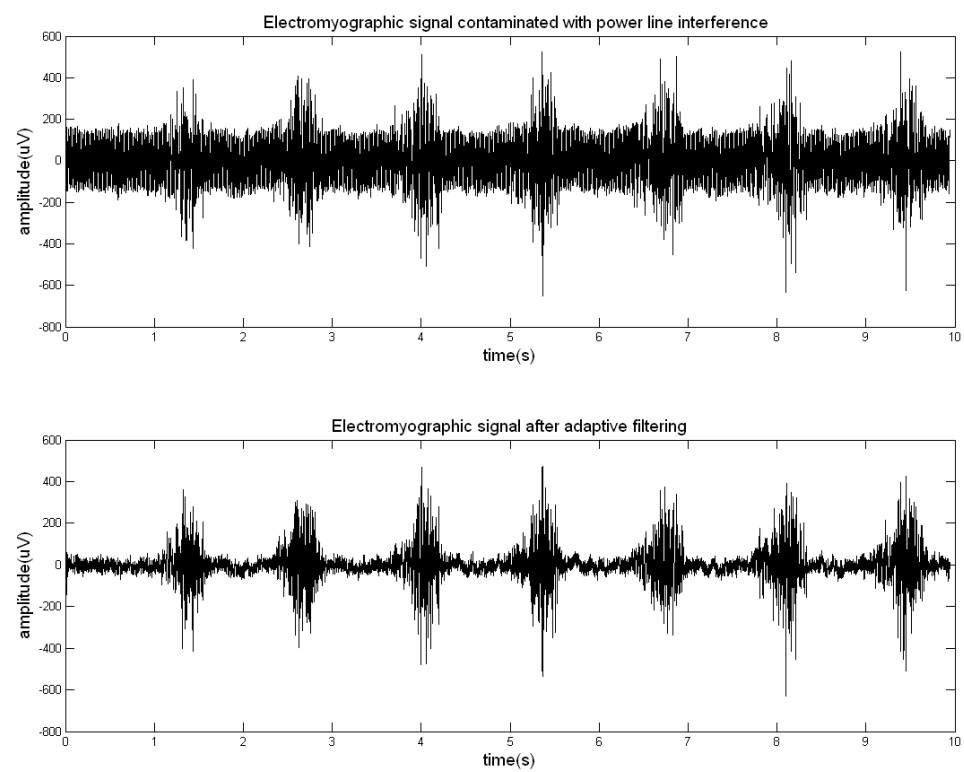

Fig. 7. Adaptive filtering performed on a SEMG signal contaminated with power-line interference

\subsection{Myoelectric Knee Joint Angle Estimation Algorithm}

Presenting the myoelectric signal directly to a classifier is impractical, because of the dimensionality and the random characteristics of the signal. It's necessary that the signal is represented as a vector with reduced dimensionality, i.e., a feature vector. The myoelectric knee angle estimation algorithm proposes the use of three stages for feature extraction and pattern classification. The first stage consists in the mixture of feature vectors from time domain and spectral analysis. A second stage will perform the reduction of the feature space, and the last stage has the goal of estimating the knee angle.

Feature Vector Extraction. Given the stochastic nature of the myoelectric signal, it can be considered as a time series, and modeled as a linear combination of their past and present values. The auto-regressive (AR) model is a convenient structure for model identification, especially when the computational speed and response time are important, as in the recognition and classification of myoelectric patterns. The autoregressive coefficients provide information about the muscular contraction. The estimate of the coefficients is performed using a recursive least squares (RLS) technique, with a forgetting factor. This method gives more weight to the most recent samples at the moment of the iteration cycle. The parameters are calculated recursively [7], as presented in equations (5), (6) and (7): 


$$
\begin{gathered}
\hat{\eta}_{k}=\hat{\eta}_{k-1}+L_{k}\left[y_{k}-\varphi_{k}^{T} \hat{\eta}_{k-1}\right] \\
P_{k}=\left[P_{k-1}-\frac{P_{k-1} \varphi_{k} \varphi_{k}^{T} P_{k-1}}{\lambda_{k} \varphi_{k}^{T} P_{k-1} \varphi_{k}}\right] \frac{1}{\lambda_{k}} \\
L_{k}=\frac{P_{k-1} \varphi_{k}}{\lambda_{k} \varphi_{k}^{T} P_{k} \varphi_{k}},
\end{gathered}
$$

where $\hat{n}_{k}$ are the vector coefficients that are estimated at discrete time $k ; \varphi_{k}$ are the regressive vectors, $P_{k}$ is the inverse correlation matrix and $L_{k}$ is the gain vector of the filter. The forgetting factor $\lambda_{k}$ controls the system response time. The coefficient estimated at instant $k$ can be interpreted as a characteristic of the SEMG signal within the time interval specified by the forgetting factor, and it is a way of determining the angular displacement that the patient wants to impose to the prosthesis [4]. The coefficients form a feature vector for the pattern classification process.

Recent research has demonstrated that a functional and efficient configuration consists of a mixture of time domain feature vectors with auto-regressive coefficients [8]. This configuration provides good classification precision, and is computationally efficient, which facilitates its implementation in embedded systems. It is also more robust to the displacement of the surface electrodes [8].

This work uses a combination of the auto-regressive model with the EMG histogram method. The EMG histogram is an extension of the zero crossing and the Willison amplitude [9]. Myoelectric signals reach relatively higher levels during the contraction process, compared to the base line amplitudes. Thus, vectors obtained from the histogram provide a measure of the frequency in which the signal reaches each level of amplitude, associated with different histogram bins. For the implementation of the histogram, the SEMG dynamic range was symmetrically subdivided into 9 bins. These bins represent intervals of amplitude in which the SEMG signal is grouped. The resulting feature vectors (auto-regressive coefficients and histogram bin counts) are concatenated, and then used as the input vector of the feature projection stage.

Feature Projection. A feature projection stage is used to reduce the dimension of the feature space of the input vectors, before the pattern classification process. This reduction is performed using an unsupervised Kohonen self-organizing map (SOM) neural network. The groups of vector coefficients obtained from each SEMG channel using the AR and histogram methods are transformed into two-dimensional vectors. With the reduction in input dimension, the SOM is able to reduce noise and absorb the large variations that appear in the original features. In addition, the SOM can shorten the training time of the supervised pattern-classification neural network. The unsupervised SOM can find the winning neuron on a 2-D map to represent the original pattern. To find the output neuron (winning node), the following steps are used, according to the learning rule of the Kohonen feature map [10]. 
Step 1: Choose random values for the initial weight vectors $W_{j}(0)$.

Step 2: Find the winning neuron $y_{c}$ at time step $t$ (similarity matching), by using the minimum-distance Euclidean criterion:

$$
y_{c}=\operatorname{argmin}\left\|x(t)-W_{j}(t)\right\|, j=1,2, \ldots, t .
$$

Step 3: Update the synaptic weight vectors of all neurons by using the following update rule:

$$
W_{j}(t+1)=W_{j}(t)+\eta(t) h_{j, y_{c}}(t)\left[x(t)-W_{j}(t)\right] .
$$

where $\eta(t)$ is the learning rate, and $h_{j, y_{c}}(t)$ is the neighbor function centered around the winner. $\eta(t)$ and $h_{j, y_{c}}(t)$ are varied dynamically during the learning stage, in order to obtain optimal results.

Step 4: Go back to step 2 until no changes in the feature map are observed.

The inputs of the Kohonen's SOM are features from each channel, and the output is the 2-D coordinate (on the $x$ and $y$ axes) on the 2-D topological net. A 2-D coordinate is a condensed feature for each channel (Fig. 8).

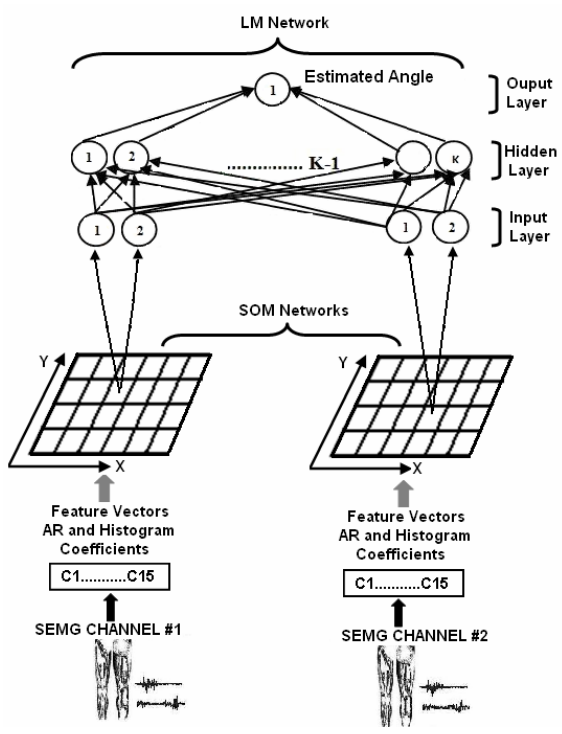

Fig. 8. Block diagram of the proposed myoelectric knee angle estimation algorithm

Myoelectric Classification. Multi-layer neural networks have been successfully applied to many difficult and nonlinear problems in diverse domains and there is considerable research on methods to accelerate the convergence time of the multilayer feedforward neural network algorithm $[11,12]$. The method used in this paper is the Levenberg-Marquardt (LM) algorithm [13], which consists in the use of the nonlinear least squares algorithm to the batch training of multi-layer perceptrons. The 
LM algorithm can be considered a modification of the Gauss-Newton method. The key step in the LM algorithm is the computation of the Jacobian matrix. The LM algorithm is very efficient when training networks which have up to a few hundred weights. Although the computational requirements of the LM algorithm become much higher after each iteration, this is fully compensated by its higher efficiency. This is especially true when high precision is required [13]. Figure 8 presents the complete block diagram of the myoelectric control algorithm.

\section{Results}

As a prototype implementation, the training and testing processes were performed in off-line mode, and the algorithms described above were implemented in Matlab. At a later stage, the full validation of the algorithm will be the executed from an embedded system running on a Linux platform.

For this demonstration, SEMG measurements were captured from four healthy subjects using $10 \mathrm{~mm} \mathrm{Ag} / \mathrm{AgCl}$ surface electrodes with conductive gel, placed on a pair of antagonistic muscles, associated with the flexion and extension movements of the knee (Fig. 3). The electrodes were arranged in bipolar configuration. The distance between the centers of the electrodes was $3-5 \mathrm{~cm}$, and the reference electrode was placed over the lateral condyle bone. Each subject was studied over the course of five days. Four 10-second measurements were performed on each day, with 5-minute rest periods between measurements. For each measurement, the subject was asked to walk in a particular direction at a constant pace. Some variability in pace was observed between measurements. The first and third measurements from each day were used for training, and the second and fourth measurements were used for testing. Thus, a total of 80 measurements were obtained, with half of them being used for training and the other half being used for testing.

For training purposes, it is essential to know information about the input and output, comparing the dimensional vectors obtained from the SOM network to the displacement angle measured with the electrogoniometer. The electrogoniometer was placed and strapped over the external side of the leg, so that it would measure the angular displacement of the knee in sagittal plane (Fig. 3c).

Figure 9 shows the estimated angle compared to the measured angle from the electrogoniometer for a representative set of signals. Although the estimated angle follows the measurement satisfactorily, the output of the LM network presents impulsive noise (Fig. 9a), which is reduced using a moving average recursive filter with a 50-sample window (Fig. 9b). This filter presents a delay of $(M-1) / 2$ samples, where $M$ is the number of averaged samples [14]. The results obtained with 50 samples of average were satisfactory, decreasing the variance and conserving the waveform's shape.

A preliminary comparison was performed between the proposed algorithm and the methods proposed by Ferreira et al. [4]. The proposed algorithm is an alternative to the approach by Ferreira et al., which consists in using the AR model for feature extraction, and a LM multi-layer perceptron neural network for pattern classification.

This quantitative comparison was based on the classification error and on the correlation coefficient. The classification error was calculated as follows: 

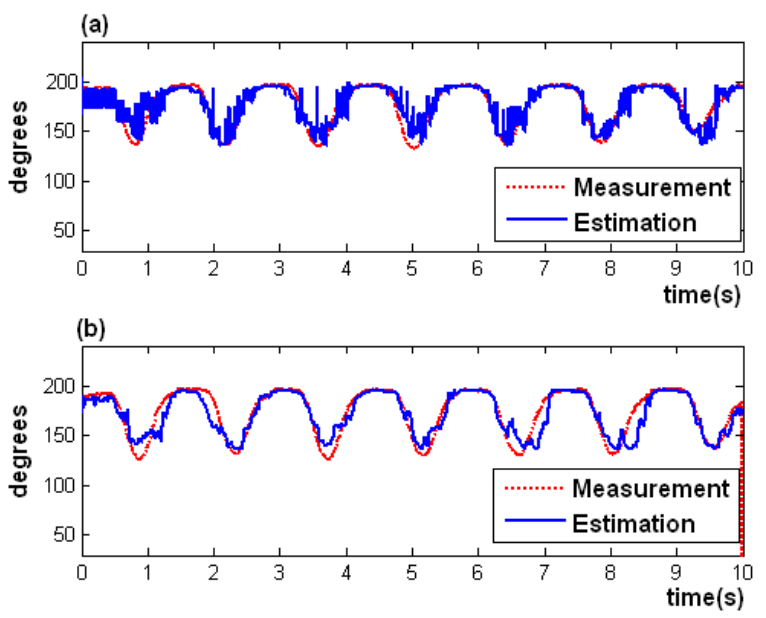

Fig. 9. Comparison of the estimated knee angle (dashed line) with the measured angle from the electrogoniometer (solid line): (a) before filtering; (b) after filtering

$$
\text { error }=\sqrt{\frac{\sum_{i=1}^{N}|x(i)-\hat{x}(i)|^{2}}{\sum_{i=1}^{N}|x(i)|^{2}}} \times 100(\%),
$$

where $x(i)$ and $\hat{x}(i)$ represent the angular values from the electrogoniometer sensor and the estimated angle values, respectively, and $N$ is the dimension of the vectors. The correlation coefficient $r$ was calculated as

$$
r=\frac{\sum_{i=0}^{N-1}\left(x_{i}-\bar{x}\right)\left(y_{i}-\bar{y}\right)}{\sqrt{\sum_{i=0}^{N-1}\left(x_{i}-\bar{x}\right)^{2} \sum_{i=0}^{N-1}\left(y_{i}-\bar{y}\right)^{2}}}
$$

where, $x_{i}$ and $y_{i}$ are the $i$-th measured and estimated joint angle value, respectively, $\bar{x}$ and $\bar{y}$ are the mean measured and estimated joint angular displacements, respectively, and $N$ is the dimension of the vectors. This coefficient provides a measurement of the degree of linear dependence between the two variables. The closer the coefficient is to either -1 or +1 , the stronger the correlation between the variables.

Table 1 presents the average classification error rate and correlation coefficient, respectively, measured in each subject's group of test signals, with the associated standard deviation. The proposed method provides moderately lower classification error and slightly higher correlation than the method by Ferreira et al. for all four subjects. 
Table 1. Comparison between the myoelectric knee joint angle estimation algorithms

\begin{tabular}{lcccc}
\hline & \multicolumn{2}{c}{ Classification error $(\%)$} & \multicolumn{2}{c}{ Correlation coefficient } \\
\cline { 2 - 5 } & Ferreira et al. & Proposed & Ferreira et al. & Proposed \\
\hline Subject A & $8.02 \pm 4.21$ & $6.56 \pm 1.85$ & $0.75 \pm 0.20$ & $0.84 \pm 0.07$ \\
Subject B & $8.18 \pm 4.70$ & $5.33 \pm 1.13$ & $0.54 \pm 0.27$ & $0.61 \pm 0.22$ \\
Subject C & $6.54 \pm 4.36$ & $5.77 \pm 3.64$ & $0.59 \pm 0.16$ & $0.59 \pm 0.90$ \\
Subject D & $6.63 \pm 3.06$ & $5.23 \pm 1.47$ & $0.71 \pm 0.17$ & $0.72 \pm 0.09$ \\
\hline
\end{tabular}

\section{Conclusions}

This paper presented the current state of development of a bioinstrumentation system for active control of leg prostheses. Features of the system and of the signal processing algorithm used in the myoelectric knee joint angle estimation algorithm were presented. The system allows the acquisition of SEMG signals with maximum amount of signal information and minimum amount of contamination from electrical noise. The results show that the system has great potential for future developments in leg prosthesis control. Preliminary analysis showed that the computational complexity of the proposed algorithm increases for each iteration during execution of the LM network. Future work will aim at optimizing the code for its execution in real time.

Acknowledgements. This work was partially supported by CAPES and CNPq.

\section{References}

1. Sommerich, C.M., Joines, S.M., Hermans, V., Moon, S.D.: Use of Surface Electromyography to Estimate Neck Muscle Activity. J. Electromyography Kinesiol 6, 377-398 (2000)

2. De Luca, C.J.: Encyclopedia of Medical Devices and Instrumentation. John G. Webster (2006)

3. Monseni-Bendpei, M.A., Watson, M.J., Richardson, B.: Application of Surface Electromyography in the Assessment of Low Back Pain: A Literature Review. J. Phys. Ther. Rev. 2, 93-105 (2000)

4. Ferreira, R.U., da Rocha, A.F., Cascão Jr., C.A., Borges, G.A., Nascimento, F.A.O., Veneziano, W.H.: Reconhecimento de Padrões de Sinais de EMG para Controle de Prótese de Perna. In: XI Congresso Brasileiro de Biomecânica (2005)

5. Asghari, M.O., Hu, H.: Myoelectric Control System - A survey. J. Biomedical Signal Processing and Control 2, 275-294 (2007)

6. Hamilton, P.S.: A comparison of Adaptive and Nonadaptive Filters for Reduction of Power Line Interference in the ECG. J. IEEE Trans. Biomed. Eng. 43, 105-109 (1996)

7. Ljung, L.: Linear System Identification. Prentice-Hall, Inc., Englewood Cliffs (1987)

8. Hargrove, L., Englehart, K., Hudgins, B.: A Training Strategy to Reduce Classification Degradation Due to Electrode Displacements in Pattern Recognition Based Myoelectric Control. J. Biomedical Signal Processing and Control 3, 175-180 (2008)

9. Zardoshti-Kermani, M., Wheeler, B.C., Badie, K., Hashemi, R.M.: EMG Feature Evaluation for Movement Control of Upper Extremity Prostheses. J. IEEE Trans. on Rehabilitation Eng. 3, 324-333 (1995) 
10. Haykin, S.: Neural Networks: A Comprehensive Foundation. Prentice Hall, New Jersey (1999)

11. Batiti, R.: First and Second Order Methods for Learning: Between Steepest Decent and Newton's Method. J. Neural Computation 4, 141-166 (1992)

12. Charalambous, C.: Conjugate Gradient Algorithm for Efficient Training of Artificial Neural Networks. IEE Circuit, Devices and System (1992)

13. Hagan, M.T., Menhaj, M.B.: Training Feedforward Networks with the Marquardt Algorithm. J. IEEE Trans. Neural Networks 5, 989-993 (1994)

14. Smith, S.W.: The Scientist and Engineer's Guide to Digital Signal Processing. California Technical Publishing, San Diego (1999) 